\title{
Interstitial defects in diamond: A quantum mechanical simulation of their EPR constants and vibrational spectra
}

Cite as: J. Chem. Phys. 153, 024119 (2020); https://doi.org/10.1063/5.0014368

Submitted: 19 May 2020 • Accepted: 24 June 2020 • Published Online: 13 July 2020

Fabio Colasuonno, (Drancesco Silvio Gentile, (D) William Mackrodt, et al.

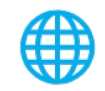

\section{ARTICLES YOU MAY BE INTERESTED IN}

Titratable Martini model for constant $\mathrm{pH}$ simulations

The Journal of Chemical Physics 153, 024118 (2020); https://doi.org/10.1063/5.0014258

The CECAM electronic structure library and the modular software development paradigm The Journal of Chemical Physics 153, 024117 (2020); https://doi.org/10.1063/5.0012901

Machine-learning approach for constructing control landscape maps of three-dimensional alignment of asymmetric-top molecules

The Journal of Chemical Physics 153, 024120 (2020); https://doi.org/10.1063/5.0012303

The Journal

SPECIAL TOPIC: Low-Dimensional

of Chemical Physics Materials for Quantum Information Science 


\title{
Interstitial defects in diamond: A quantum mechanical simulation of their EPR constants and vibrational spectra
}

\author{
Cite as: J. Chem. Phys. 153, 024119 (2020); doi: 10.1063/5.0014368 \\ Submitted: 19 May 2020 - Accepted: 24 June 2020 • \\ Published Online: 13 July 2020
}

\begin{abstract}
Fabio Colasuonno,' (D) Francesco Silvio Gentile, ${ }^{1, a)}$ (D) William Mackrodt, ${ }^{\prime}$ (D) Anna Maria Ferrari, Alexander Platonenko, ${ }^{2}$ (D) and Roberto Dovesi ${ }^{1}$
\end{abstract}

\author{
AFFILIATIONS \\ ${ }^{1}$ Dipartimento di Chimica, Università di Torino and NIS (Nanostructured Interfaces and Surfaces) Centre, \\ Via P. Giuria 5, 10125 Torino, Italy \\ ${ }^{2}$ Institute of Solid State Physics, University of Latvia, 8 Kengaraga Street, LV1063 Riga, Latvia
}

\begin{abstract}
a) Author to whom correspondence should be addressed: francesco.gentile@unina.it
\end{abstract}

\begin{abstract}
The local geometry, electronic structure, and vibrational features of three vicinal double interstitial defects in diamond, $\mathrm{I}_{C} \mathrm{I}_{C}, \mathrm{I}_{C} \mathrm{I}_{N}$, and $\mathrm{I}_{N} \mathrm{I}_{N}$, are investigated and compared with those of three "simple" $\langle 100\rangle$ interstitial defects, $\mathrm{I}_{C C}, \mathrm{I}_{C N}$, and $\mathrm{I}_{N N}$, previously reported by Salustro et al. [Phys. Chem. Chem. Phys. 20, 16615 (2018)], using a similar quantum mechanical approach based on the B3LYP functional constructed from Gaussian-type basis sets, within a supercell scheme, as implemented in the CRYSTAL code. For the first time, the Fermi contact term and hyperfine coupling tensor $\mathcal{B}$ of the four open shell structures, $\mathrm{I}_{C} \mathrm{I}_{C}, \mathrm{I}_{C} \mathrm{I}_{N}, \mathrm{I}_{C C}$, and $\mathrm{I}_{C N}$, are evaluated and compared with the available experimental EPR data. For the two double interstitial defects, the agreement with experiment is good, whereas that for the single interstitials is found to be very poor, for which a likely reason is the incorrect attribution of the EPR spectra to uncertain atomic details of the microstructure of the samples. The infrared spectra of the three double interstitial defects exhibit at least two peaks that can be used for their characterization.
\end{abstract}

Published under license by AIP Publishing. https://doi.org/10.1063/5.0014368

\section{INTRODUCTION}

Natural and synthetic diamonds have attracted significant interest for many possible applications, such as high temperature diodes, transistors, thermistors, detectors, and windows for plasma heating in nuclear fusion power plants, ${ }^{2-4}$ in view of their high melting point, thermal conductivity, hardness, and wide bandgap. The presence of intrinsic and extrinsic point defects in the crystalline lattice can dramatically modify some of the above properties so that over the last few decades, the investigation of both native and induced defects in diamond has been of continuing interest both from experimental and computational perspectives. ${ }^{5-1}$ More recently, point defects in diamond have come into even sharper focus with the emergence and development of quantum dot devices based on diamond. ${ }^{13,14}$ Among the intrinsic defects, the vacancy $\mathrm{V}^{15-18}$ and $\langle 100\rangle$ self-interstitial (see Fig. 1, top left, for the latter $)^{8,19-21}$ are the most common, and their formation and recombination mechanisms have been investigated. ${ }^{22,23}$ Of the possible impurities, nitrogen is by far the most common, in both synthetic and natural diamonds, with concentrations of thousands of parts per million (ppm) not uncommon. ${ }^{24}$ One of the reasons for this is that nitrogen can be hosted relatively easily in the diamond lattice as a result of its atomic radius, which is very close to that of carbon. However, despite this, substitutional nitrogen, $\mathrm{N}_{s}$, can lead to the displacement of a $\mathrm{C}$ atom to form a $\langle 100\rangle$ interstitial as a result of the difference in the electronic configurations of $\mathrm{C}$ and $\mathrm{N}$, where $\mathrm{N}_{s}$ is one of the two three-coordinated atoms of the interstitial. Thus, in the presence of nitrogen, three kinds of $\langle 100\rangle$ interstitial defect (indicated here as $\mathrm{I}_{x y}$, where $x$ and $y$ are the two threefold coordinated atoms) can, in principle, be found, namely, $\mathrm{I}_{C C}, \mathrm{I}_{C N}$, and $\mathrm{I}_{N N}$. Furthermore, two $\mathrm{I}_{x y}$ defects can interact to generate a more stable structure, in which two three-coordinated atoms and two four-coordinated atoms form approximately square, planar structures (see Fig. 2), referred to 

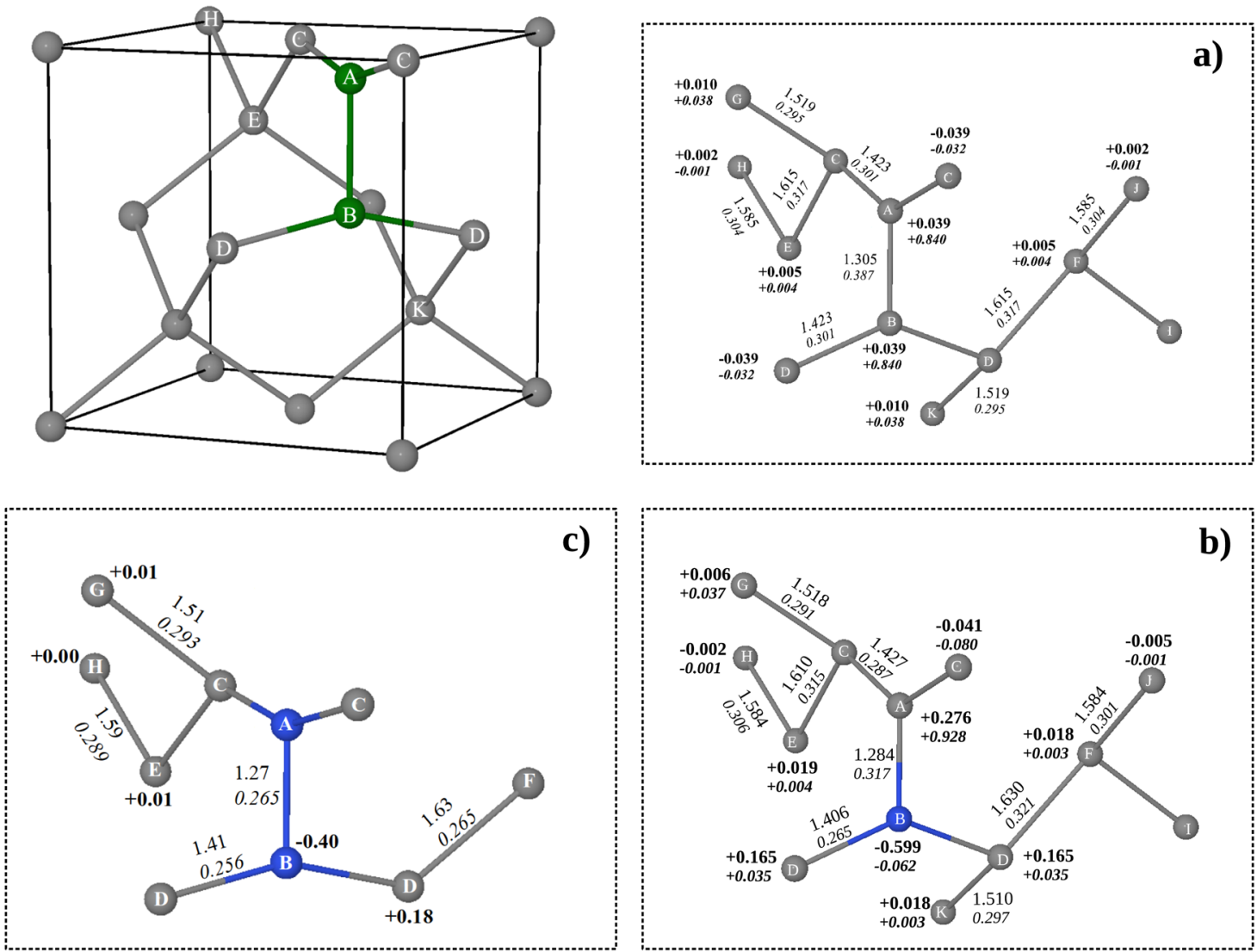

FIG. 1. Top left: The conventional diamond cell and interstitial $I_{x y}$ defect (defect atoms in green). Panels (a), (b), and (c) refer to $I_{C C}, I_{C N}$, and $I_{N N}$, with nitrogen atoms in blue. Four numbers are shown in the schemes. Near the atoms, the atomic net charges $q$ (top) and the magnetic moments $\mu$ (bottom) are shown. Along the bonds, the atom-atom distances $\mathrm{R}$ (top) and the bond populations $b$ (bottom) are reported. $Q$ and $\mu$ are in bold. $b$ and $\mathrm{R}$ are in italic. $q, \mu$, and $b$, as resulting from a Mulliken analysis, are given in $|e| . R$ is in $\AA$.

hereafter as $I_{x} I_{y}$. The two three-coordinated atoms are not first neighbors, as is the case for $\mathrm{I}_{x y}$, which is probably the main reason for the higher stability of $\mathrm{I}_{x} \mathrm{I}_{y}$ with respect to $\mathrm{I}_{x y}$.

Relating experimental IR, Raman, or EPR spectra to a specific defect or a defect structure is fraught with difficulty and hence challenging, for it is essential to take into account the presence of other possible defects with potentially overlapping spectra. Computer simulations, on the other hand, are not subject to such difficulties, for the particular defect or defects in question are specified exactly a priori, in terms of their charge, spin, and multiplicity, if not the precise details of the defective lattice, so that there is always a 1:1 relationship between the defect and the computed spectra. Depending on the level of agreement with observation, these can then be used to validate, or otherwise, structures that have either been derived from or ascribed to the experimental spectra.

The purpose of the present paper, then, is twofold: the first is to report the predicted structures and EPR, IR, and Raman spectra of $\mathrm{I}_{x} \mathrm{I}_{y}$ and $\mathrm{I}_{x y}$ defects obtained from quantum mechanical simulations and assess whether collectively or separately the spectra are sufficiently distinct for them to uniquely characterize the individual defects; the second is to compare the computed and observed spectra, where available, and, in particular, the computed structures with those inferred from or ascribed to the spectra.

Only four of the six defects $\left(\mathrm{I}_{C} \mathrm{I}_{C}, \mathrm{I}_{C} \mathrm{I}_{N}, \mathrm{I}_{C C}\right.$, and $\left.\mathrm{I}_{C N}\right)$, have neutral, open shell ground states and, hence, are EPR active. It is interesting to note, therefore, that four sets of EPR absorptions have been tentatively attributed to these defects, namely, $\mathrm{I}_{C} \mathrm{I}_{C}\left(\mathrm{R} 1^{25,26}\right)$, $\mathrm{I}_{C C}\left(\mathrm{R} 2^{25}\right), \mathrm{I}_{C} \mathrm{I}_{N}\left(\mathrm{WAR} 10^{27}\right)$, and $\mathrm{I}_{C N}\left(\mathrm{WAR} 9^{27}\right)$.

All six compounds are IR and Raman active and could, therefore, in principle, be used to identify each of the defects.

In the present paper, all-electron quantum mechanical calculations based on the B3LYP functional utilizing Gaussian basis sets, and formulated within a supercell scheme, ${ }^{28,29}$ have been used to study these six interstitial defects. This same approach has been used previously to investigate the structural and vibrational features of the three single interstitial defects, $\mathrm{I}_{x y}{ }^{1,30}$ Here, we pay particular attention to the EPR spectra for $\mathrm{I}_{C} \mathrm{I}_{C}, \mathrm{I}_{C} \mathrm{I}_{N}, \mathrm{I}_{C C}$, and $\mathrm{I}_{C N}$ and compare the computed data with experiment.

Recent applications to various defects (substitutional $\mathrm{N}$, or $\mathrm{N}_{s},{ }^{31} \mathrm{VN}_{2}^{-},{ }^{32} \mathrm{~N}_{2}^{+33}$ ) have confirmed that the present approach is able 

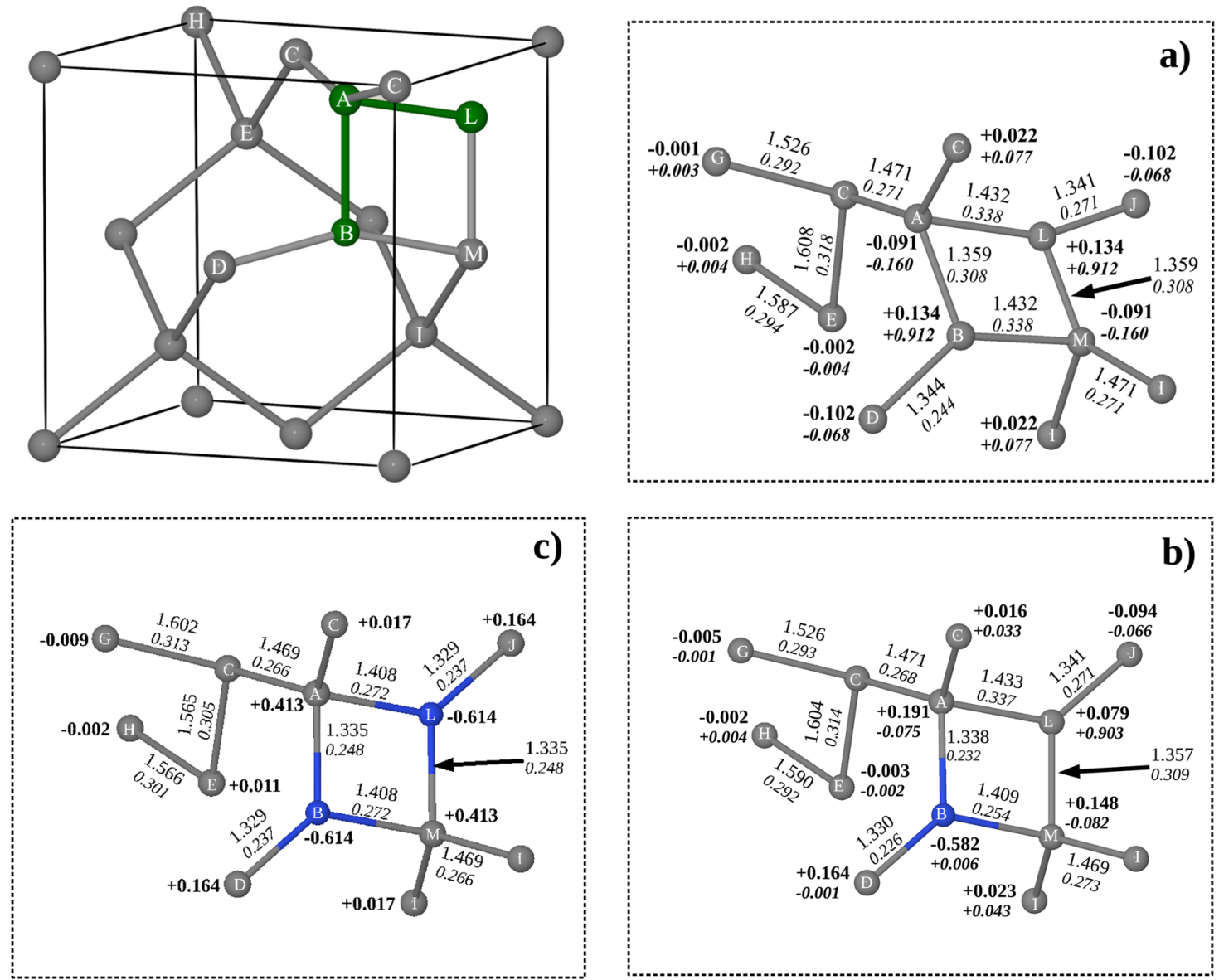

FIG. 2. Top left: The conventional diamond cell and interstitial $I_{x} I_{y}$ defect (defect atoms in green). Panels (a), (b), and (c) refer to $I_{C} I_{C}$, $I_{C} I_{N}$, and $I_{N} I_{N}$, respectively. Nitrogen atoms are in blue. Symbols and other information as in Fig. 1.

to provide an excellent agreement with the experimental EPR results, when the latter have been accurately determined.

This paper is structured as follows: Sec. II describes the computational methods used in this study. Section III contains the results and is organized in three parts. Subsection III A is devoted to the equilibrium geometries, charge and spin distributions, and band structures. Subsection III B reports the computed hyperfine coupling tensors and compares them with the available experimental data, while Subsection III C describes the vibrational features of these defects; finally, Sec. IV discusses the possible identification of the six $\mathrm{I}_{x y}$ and $\mathrm{I}_{x} \mathrm{I}_{y}$ defects through their EPR and/or IR spectra and draws some tentative conclusions.

\section{COMPUTATIONAL METHODS}

Calculations have been performed by using the B3LYP global hybrid functional ${ }^{34,35}$ based on Pople's all electron $6-21 \mathrm{G}^{36}$ basis sets of Gaussian-type functions, except for the exponents of the most diffuse sp shells of carbon and nitrogen, for which values of $0.23 \mathrm{bohr}^{-2}$ and $0.30 \mathrm{bohr}^{-2}$ have been adopted. However, a much richer (and more expensive) basis set, ${ }^{37}$ containing $6 s$ shells, with 811111 contractions of primitive functions, $2 p$ shells, with 41 contractions, and $1 d$ shell, with a total 17 atomic orbitals (AOs) per atom, has been adopted for the EPR calculations. This compares with the 9 AOs/atom of the smaller basis set. The enlarged set was originally derived from the standard 6-31G version, and although it was later extended, it is here referred to as $6-31 \mathrm{G}-\mathrm{J}^{*}$. It was designed to better describe the core electrons, but the valence shells are also richer, with exponents of the most diffuse $\mathrm{s}$ and $\mathrm{p}$ functions of $\mathrm{C}$ and $\mathrm{N}$ set to $0.169 \mathrm{bohr}^{-2}$ and 0.212 bohr $^{-2}$, respectively. ${ }^{37}$

This combination of the B3LYP functional and the $6-31 \mathrm{G}-\mathrm{J}^{*}$ basis set has been shown to provide accurate results for the EPR data of three defects in diamond, namely, $\mathrm{N}_{s},{ }^{31} \mathrm{VN}_{2}^{-}$(the negatively charged form of $\mathrm{VN}_{2}$, with two nitrogen atoms around a vacancy), ${ }^{32}$ and $\mathrm{N}_{2}^{+}$, the positively charged form of the so called A defect (two vicinal substitutional $\mathrm{N}$ atoms), ${ }^{33}$ where in all three cases, the spin state is a doublet.

The Coulomb and exchange infinite lattice series are controlled by five parameters, $\mathrm{T}_{i}$, which have been set to $8\left(\mathrm{~T}_{1}-\mathrm{T}_{4}\right)$ and 16 $\left(\mathrm{T}_{5}\right)$. The convergence threshold on the SCF energy was set to 
$10^{-8}$ hartree for structural optimizations and to $10^{-10}$ hartree for vibration frequency calculations. ered.

A cubic supercell containing $216\left(\mathrm{~S}_{216}\right)$ atoms has been consid-

The DFT exchange-correlation contribution and its gradient were evaluated by numerical integration over the unit cell volume. The generation of the integration grid points was based on an atomic partition method, originally developed by Becke ${ }^{38}$ for molecules and further extended to periodic systems. ${ }^{28,29}$ Within this scheme, the unit cell is partitioned into atomic volumes centered on the nuclei, where each point has an associated weight. The radial and angular points for the integration grid are generated using a Gauss-Legendre quadrature and Lebedev two-dimensional distribution, respectively. The choice of a suitable grid is crucial both for numerical accuracy and the optimization of computational resources. In this study, a pruned grid with 75 radial and 974 angular points has been used, whose accuracy can be measured by comparing the integrated charge density of $N_{i}=1309.016$ for $\mathrm{I}_{C} \mathrm{I}_{N}$, with the total number of 1309 electrons in the unit cell. A $\Gamma$-point centered Pack-Monkhorst grid $^{39}$ for sampling reciprocal space has been used, consisting of $2 \times 2 \times 2=8\left(\mathrm{~S}_{216}\right)$ k-points in the first Brillouin Zone. Harmonic phonon frequencies, $\omega_{p}$, at the $\Gamma$ point, the only point that contributes to the IR spectrum, were obtained from the diagonalization of the mass-weighted Hessian matrix of the second energy derivatives with respect to atomic displacements $u{ }^{40}$

$$
W_{a i, b j}^{\Gamma}=\frac{H_{a i, b j}^{0}}{\sqrt{M_{a} M_{b}}} \quad \text { with } \quad H_{a i, b j}^{0}=\left(\frac{\partial^{2} E}{\partial u_{a i}^{\mathbf{0}} \partial u_{b j}^{\mathbf{0}}}\right),
$$

where atoms $a$ and $b$ (with atomic masses $M_{a}$ and $M_{b}$ ) in the reference cell, $\mathbf{0}$, are displaced along the $i$ th and $j$ th Cartesian directions, respectively. First-order derivatives were computed analytically, whereas second order derivatives were obtained numerically, using a two-point formula based on the difference between the gradient at the equilibrium position and that after a displacement of 0.003 bohr, along each Cartesian coordinate. It is important to note that as the optimization of the structure is a numerical process, the gradient at equilibrium is not exactly null but simply lower than the selected thresholds of the optimizer. Integrated intensities for IR absorption $\mathcal{I}_{p}$ are computed for each mode $p$ using a computational scheme ${ }^{41}$ based on the Berry phase, ${ }^{42}$ wherein the derivatives of the dipole moment with respect to the Cartesian coordinates of the unit cell atoms are evaluated numerically in a similar way to that used for the numerical derivative of the energy gradient in obtaining the Hessian matrix. The IR spectrum is then computed from the $\Gamma$-point transverse-optic (TO) modes and the adoption of a pseudo-Voigt functional form consisting of Lorentzian curves with a full width at half maximum of $8 \mathrm{~cm}^{-1}$.

The coupling between the unpaired electron spin (S) and the system of the nuclear spins (I) is described through the spin Hamiltonian

$$
H=\sum_{n} \mathbf{S} \cdot \mathcal{A}^{n} \cdot \mathbf{I}^{n},
$$

where $\mathbf{I}^{n}$ and $\mathcal{A}^{n}$ refer to the nuclear spin and hyperfine coupling tensor related to the $n$th nucleus, at site $\mathbf{R}_{n}$, respectively. $\mathcal{A}^{n}$ can be written in the form

$$
\mathcal{A}^{n}=A_{\text {iso }}^{n} \cdot I+\mathcal{B}^{n},
$$

where $I$ is a $3 \times 3$ identity matrix, or

$$
A_{i j}^{n}=A_{i s o}^{n} \delta_{i j}+B_{i j}^{n},
$$

where $A_{i s o}^{n}$ is the isotropic contribution to $\mathcal{A}^{n}$, often referred to as the Fermi contact term, and $\mathcal{B}^{n}$ is the anisotropic dipole-dipole interaction of the electron and nuclear spins. The Fermi contact term for $\mathrm{n}$ unpaired electrons of total spin $S_{z}$,

$$
A_{i s o}^{n}=g_{e} \mu_{e} g_{n} \mu_{N} \frac{4 \pi}{3 S_{z}}\left|\psi\left(\mathbf{R}_{n}\right)\right|^{2}
$$

relates to the direct interaction of nuclear and electron spins and is only non-zero for states with finite electron spin density, $\left|\psi\left(\mathbf{R}_{n}\right)\right|^{2}$, at nuclear sites $\mathbf{R}_{n}$, namely, those with unpaired electrons in $s$-subshells with $S$ the spin state of the system. $g_{e}, \mu_{e}, g_{n}$, and $\mu_{N}$ are the freeelectron $g$-factor, Bohr magneton, gyromagnetic ratio of $\mathbf{I}^{n}$, and nuclear magneton, respectively. The elements of the traceless tensor $\mathcal{B}^{n}$ at nucleus $\mathbf{R}_{n}$ are defined by

$$
B_{i j}^{n}=g_{e} \mu_{e} g_{n} \mu_{N} \frac{1}{2 S_{z}} \int d \mathbf{r}_{n}\left|\psi\left(\mathbf{r}_{n}\right)\right|^{2}\left(\frac{3 \mathbf{r}_{n i} \mathbf{r}_{n j}-\left|\mathbf{r}_{n}\right|^{2} \delta_{i j}}{\left|\mathbf{r}_{n}\right|^{5}}\right),
$$

where $\mathbf{r}_{n}$ is the distance to $\mathbf{R}_{n}$. $\mathcal{B}^{n}$ is usually written in terms of its three (principal axes) eigenvalues, $B_{1}^{n}, B_{2}^{n}$, and $B_{3}^{n}$, and is a measure of the unpaired electron populations of valence $p$ and $d$ orbitals centered on the magnetic nucleus and of all orbitals centered on neighboring atoms. Clearly, $A_{i s o}^{n}=\left(A_{1}^{n}+A_{2}^{n}+A_{3}^{n}\right) / 3$ since $\mathcal{B}^{n}$ is traceless, and, for a nuclear site with axial symmetry, we have $A_{\text {iso }}^{n}=\left(A_{\|}^{n}+2 A_{\perp}^{n}\right) / 3$ and $B_{\|}^{n}+2 B_{\perp}^{n}=0$.

When the local point symmetry of an atom is sufficiently low, the $\mathcal{B}^{n}$ tensor is not diagonal. Diagonalization, then, generates the eigenvalues, referred to above, and the eigenvectors that define the orientation of the $\mathcal{B}^{n}$ principal axes with respect to the Cartesian frame. This relative orientation is usually expressed in terms of the angles $\theta$ and $\varphi$ formed by the largest $\mathbf{B}$ eigenvector and the $\langle 100\rangle$ and $\langle 001\rangle$ crystallographic directions, respectively.

An important point to note, especially with regard to electronic structure calculations, is that $A_{i s o}^{n}$ and $B_{i j}^{n}$ impose different requirements on the wavefunction, for whereas $A_{i s o}^{n}$ is determined by the electron density at the nuclear positions, as indicated in Eq. (5), $B_{i j}^{n}$ samples the long-range properties of the wavefunction as a result of the $r^{-3}$ scaling in the integrand in Eq. (6). It is essential, therefore, for basis sets to be sufficiently flexible so that these differing dependencies can be satisfied.

\section{RESULTS}

\section{A. Geometry, charge distribution, formation energy, and band structure}

The electronic, structural, and vibrational properties of $\mathrm{I}_{x y}$ defects have been investigated previously ${ }^{1,30}$ using the same basis set, DFT functional, computational conditions, and computer code (CRYSTAL). Here, we summarize the main results of this study, both for the purposes of comparison with the present results and to extend the analysis to the EPR constants, which were not investigated therein. 
In the case of the single (split $\langle 001\rangle$ ) interstitial defects, the two interstitial atoms are three-coordinated ${ }^{43}$ so that in the case of $\mathrm{I}_{N N}$, the ground state is a closed shell, with a covalent bond between the two $\mathrm{N}$ atoms at an internuclear distance of $1.27 \AA$ and a Mulliken bond population of $+0.265|e|$. The net charge on $\mathrm{N}$ is $-0.40|e|$, which is nearly exactly compensated by charges of $+0.18 \times 2|e|$ at atoms D and C (see Fig. 1). The lone pairs of the two nitrogen atoms point in an orthogonal direction to the ACC and BDD planes, respectively.

In $\mathrm{I}_{C N}$, the defective carbon atom contains an unpaired electron, with a calculated magnetic moment, $\mu$, of $+0.928|e|$, so that the ground state is a doublet $\left(\mathrm{S}_{z}=1 / 2\right)$ and hence EPR active. The N-C distance is $1.28 \AA$, leading to a strong covalent bond (bond population $+0.317|e|)$ and net charges of $+0.276|e|$ and $-0.599|e|$ on $\mathrm{C}$ and $\mathrm{N}$, respectively, while the charge on atom $\mathrm{D}$, which is first neighbor to $\mathrm{N}$, is $+0.17|e|$. Overall, the perturbation of the charge distribution, from that in pristine diamond, is found to be very local.

In terms of spin pairing alone, the unpaired electrons on the two defect carbon atoms in $\mathrm{I}_{C C}$ might seem suitable for bond formation. However, as shown in Fig. 1, the ACC and BDD planes, which contain the two unpaired electrons, are orthogonal so that covalent coupling cannot occur. Despite the short internuclear separation of $1.30 \AA$, the interaction between the two unpaired electrons is very weak, giving rise to a singlet state, which is more stable than the triplet by only $0.027 \mathrm{eV}(313 \mathrm{~K})$. The magnetic moments of the two spin states, $\mu$, are identical $(0.840|e|)$ and the bond population, $+0.387|e|$, indicates a strong covalent bond. The net charge $\mathrm{q}$ on the two three-coordinated carbon atoms is $+0.039|e|$.

The large difference in polarity of the $\mathrm{CN}$ and $\mathrm{CC}$ bonds has important consequences for the intensity of the active IR modes, as shown below.

The top left panel of Fig. 2 shows the conventional diamond lattice containing the double interstitial defect. The four atoms involved in the defect are indicated as A, B, L, and $\mathrm{M}$ : A and $\mathrm{M}$ are in all cases carbon atoms and are fourfold coordinated; $\mathrm{B}$ and $\mathrm{L}$ are threefold coordinated and can be both $\mathrm{N}\left(\mathrm{I}_{N} \mathrm{I}_{N}\right)$, both $\mathrm{C}\left(\mathrm{I}_{C} \mathrm{I}_{C}\right)$, or one $\mathrm{C}$ and one $\mathrm{N}\left(\mathrm{I}_{C} \mathrm{I}_{N}\right)$. $\mathrm{N}$ atoms are shown in blue. It is likely that two of the defects, $\mathrm{I}_{C} \mathrm{I}_{N}$ and $\mathrm{I}_{C} \mathrm{I}_{C}$, were detected by Felton et al., Twitchen et al., ${ }^{26}$ and Hunt et al., ${ }^{25}$ where they were named WAR10 and $\mathrm{R} 1$, respectively. In the case of $\mathrm{I}_{N} \mathrm{I}_{N}$, on the other hand, we were unable to find any experimental evidence for its existence.

The equilibrium geometry of the three defects is shown in panels (a), (b), and (c) of Fig. 2.

As in the case of the single interstitial, when the two defect atoms, $\mathrm{B}$ and $\mathrm{L}$, are nitrogen, the ground state is a closed shell; when one atom is $\mathrm{C}$ and the other is $\mathrm{N}$ [atom L in panel (b) of Fig. 2], the ground state is a doublet, with a single unpaired electron $(\mu$ $=+0.903|e|)$; finally, when $\mathrm{B}$ and $\mathrm{L}$ are both carbon atoms, the two unpaired electrons can form a triplet $\left(\mathrm{S}_{z}=1\right)$, which is shown in panel (a) of Fig. 2, or an open shell singlet $\left(\mathrm{S}_{z}=0\right)$, where the former is found to be the more stable by $0.034 \mathrm{eV}$. The open shell nature of the singlet is reflected in the magnetic moment, $\mu(+0.912|e|)$, which is identical to that in the triplet state. The reason for the reversed order of stability of the singlet and triplet states in $\mathrm{I}_{C C}$ and $\mathrm{I}_{C} \mathrm{I}_{C}$ lies in the much shorter distance between the sites containing the unpaired electrons in $\mathrm{I}_{C C}$, for at shorter distances, i.e., greater confinement, the singlet state is always favored over the triplet by virtue of the reduced Pauli repulsion between electrons of opposite spin.
Spin density maps in planes containing the interstitial atoms in $\mathrm{I}_{C} \mathrm{I}_{N}$ and $\mathrm{I}_{C} \mathrm{I}_{C}$ are shown in Fig. 4, in which the very strong spin localization at the three-coordinated carbon atom(s), the low spin density at $\mathrm{N}$, and the spin polarization of the first neighbors of the defect atoms are clearly evident.

The formation energies, $\mathrm{E}_{f}^{i}$, of the three double interstitial defects are reported in Table I. For future and cross comparison, we provide three definitions of $\mathrm{E}_{f}$, identified by the superscript, $i$, although only the third one is briefly discussed here. A systematic discussion of the mechanism and energetic of the defect formation is beyond the scope of the present paper, mainly focused on the identification of the defects through the EPR signal.

$\mathrm{E}_{f}^{1}$ and $\mathrm{E}_{f}^{2}$ are defined through the following reaction:

$$
\text { Diamond }+b \cdot \mathrm{B}+l \cdot \mathrm{L} \rightarrow \mathrm{I}_{B} \mathrm{I}_{L} .
$$

Atoms $\mathrm{B}$ and $\mathrm{L}$ can be both $\mathrm{C}$ or $\mathrm{N}$ with the restriction that $b+l=2 . \mathrm{E}_{f}^{1}$ is calculated with reference to the isolated $\mathrm{C}$ and $\mathrm{N}$ atoms, with energies of $-38.0559 \mathrm{E}_{h}$ and $-54.5228 \mathrm{E}_{h}$, respectively, while $\mathrm{E}_{f}^{2}$ is calculated with respect to $1 / 2 \mathrm{~N}_{2}$ and the $\mathrm{C}$ atom in diamond, where the reference energies are $-37.8106 \mathrm{E}_{h}$ and $-54.6563 \mathrm{E}_{h} . \mathrm{E}_{f}^{3}$ is the formation energy of the double interstitial defects, which is computed as $\mathrm{E}_{f}^{3}=\mathrm{E}\left(I_{x} I_{y}\right)-\mathrm{E}\left(I_{x y}^{1}\right)-\mathrm{E}\left(I_{x y}^{2}\right)$, where $I_{x y}^{1}$ and $I_{x y}^{2}$ are defined in columns 5 and 6 of the table. The energies of the single interstitial defects are $-8257.7081 \mathrm{E}_{h},-8274.3809 \mathrm{E}_{h}$, and $-8252.9898 \mathrm{E}_{h}$ for $\mathrm{I}_{C C}, \mathrm{I}_{C N}$, and $\mathrm{I}_{N N}$, respectively. In every case, the $\mathrm{I}_{x}-\mathrm{I}_{y}$ defects are more stable than the two constituent defects, $\mathrm{I}_{x y}$, by about $6 \mathrm{eV}$. This is supported by the experimental observation that to obtain double interstitial defects, it is sufficient to reach high concentrations of single interstitial defects in the host lattice. This is not surprising since combining two defects, each with two threefold coordinated atoms, results in a single defect with only two three-coordinated atoms.

The band structures of the three $\mathrm{I}_{x}-\mathrm{I}_{y}$ defects are collected in Fig. 3, where the continuous black and dotted red lines indicate $\alpha$ and $\beta$ bands, respectively, and the horizontal blue line represents the Fermi level $\left(\mathrm{E}_{F}\right)$. For the closed-shell system, $\mathrm{I}_{N} \mathrm{I}_{N}$, the $\alpha$ and $\beta$ bands coincide. We note that the gap of perfect diamond, derived from the present functional and basis set, is $5.73 \mathrm{eV}$, which compares favourably with the experimental value of $5.80 \mathrm{eV} .{ }^{44}$ In all three panels, energy differences refer to the $\Gamma$ point, while the vertical excitations from the highest occupied defect levels to the lower edge of the conduction band (CBLE) are indicated by orange arrows. In the case of the $\mathrm{I}_{N} \mathrm{I}_{N}$ (right panel of Fig. 3), the energies of the two lone

TABLE I. Formation energies (in $\mathrm{eV}$ ) of the $\mathrm{I}_{x}-\mathrm{I}_{y}$ defects, computed with reference to the isolated atoms $\left(\mathrm{E}_{f}^{1}\right)$, to the $\mathrm{N}_{2}$ molecule and diamond $\left(\mathrm{E}_{f}^{2}\right)$, or to the combination of two interstitial defects $\mathrm{I}_{x y}^{1}$ and $\mathrm{I}_{x y}^{2}\left(\mathrm{E}_{f}^{3}\right)$. The total energy of the corresponding pristine diamond supercell is $-8220.07866 \mathrm{E}_{h}\left(-37.8106 \mathrm{E}_{h}\right.$ per carbon atom). The total energy, in $E_{h}$, of the defective $S_{216}$ supercell is also reported.

\begin{tabular}{lrccccc}
\hline \hline Defect & Total energy & $\mathrm{E}_{f}^{1}$ & $\mathrm{E}_{f}^{2}$ & $\mathrm{I}_{x y}^{1}$ & $\mathrm{I}_{x y}^{2}$ & $\mathrm{E}_{f}^{3}$ \\
\hline $\mathrm{I}_{\mathrm{C}} \mathrm{I}_{\mathrm{C}}$ & -8295.55220 & 17.37 & 4.02 & $\mathrm{I}_{\mathrm{CC}}$ & $\mathrm{I}_{\mathrm{CC}}$ & -5.84 \\
$\mathrm{I}_{\mathrm{C}} \mathrm{I}_{\mathrm{N}}$ & -8312.23266 & 15.19 & 5.03 & $\mathrm{I}_{\mathrm{CC}}$ & $\mathrm{I}_{\mathrm{CN}}$ & -6.04 \\
$\mathrm{I}_{\mathrm{N}} \mathrm{I}_{\mathrm{N}}$ & -8328.91316 & 13.01 & 6.05 & $\mathrm{I}_{\mathrm{CN}}$ & $\mathrm{I}_{\mathrm{CN}}$ & -6.26 \\
\hline \hline
\end{tabular}



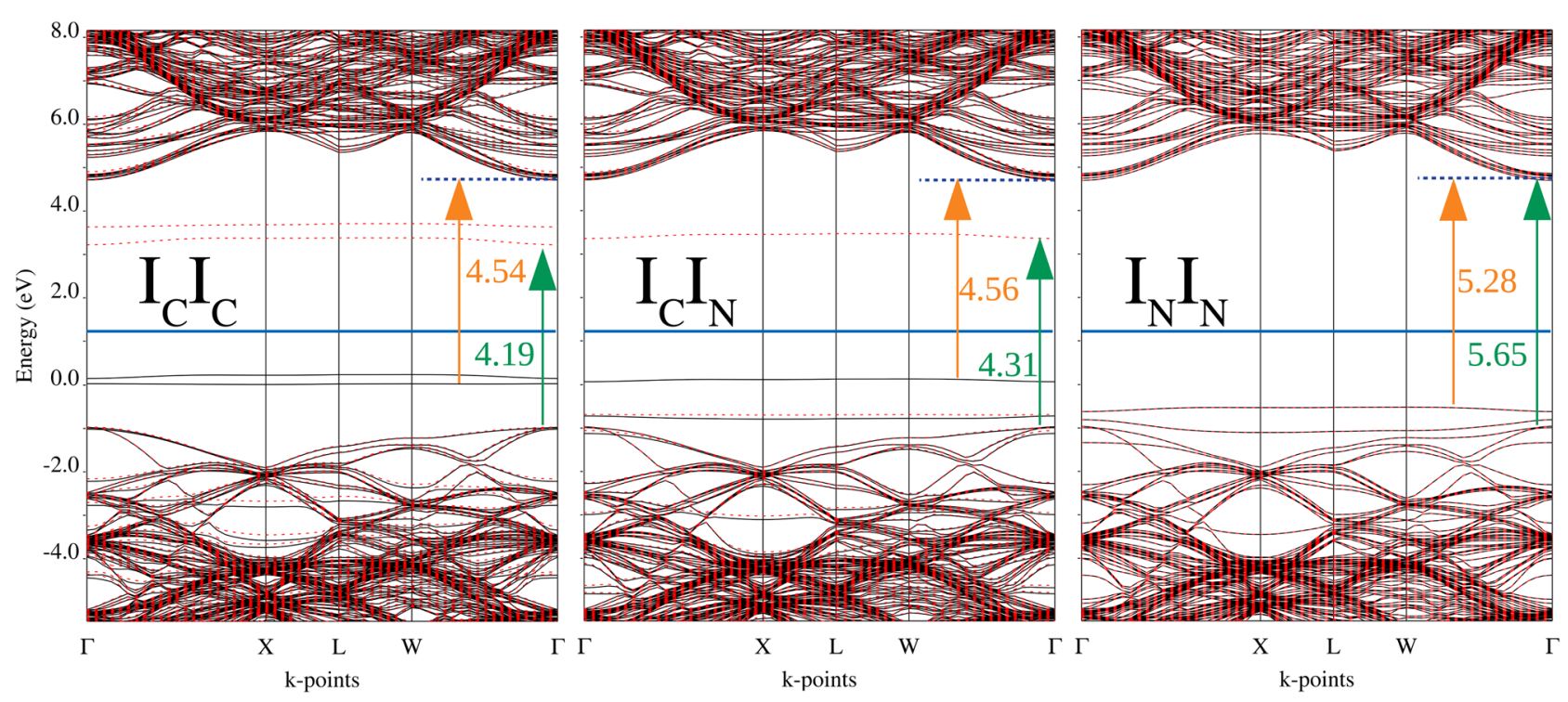

FIG. 3. Band structures of $I_{C} I_{C}$ (left), $I_{C} I_{N}$ (central), and $I_{N} I_{N}$ (right). The blue line represents the Fermi energy level. Continuous and dashed lines indicate $\alpha$ and $\beta$ bands, respectively. Vertical arrows indicate the energy difference between the top of the valence band and the lowest unoccupied defect level (green) and between the lowest occupied defect level and the bottom of the conduction band (orange), both at the $\Gamma$ point.

pairs of the nitrogen atoms are seen to be marginally above the top of the valence band (TVB) of the perfect system $(0.34 \mathrm{eV}$ and $0.15 \mathrm{eV})$ and the corresponding antibonding states, at the CBLE, leading to a vertical energy difference of $5.25 \mathrm{eV}$. The energy difference between the top of the valence band (TVB) and the lowest unoccupied state is shown in green, where the value, $5.65 \mathrm{eV}$, is $0.08 \mathrm{eV}$ smaller than the gap in pristine diamond. This lowering is due to the presence of one of the antibonding states of the nitrogen lone pairs.

In $\mathrm{I}_{C} \mathrm{I}_{N}$ (central panel), the doubly occupied lone pair band of $\mathrm{N}$ lies $0.27 \mathrm{eV}$ above the TVB, while the (singly occupied) spin-band of the unpaired electron of the $\mathrm{C}$ atom is further $0.76 \mathrm{eV}$ above at
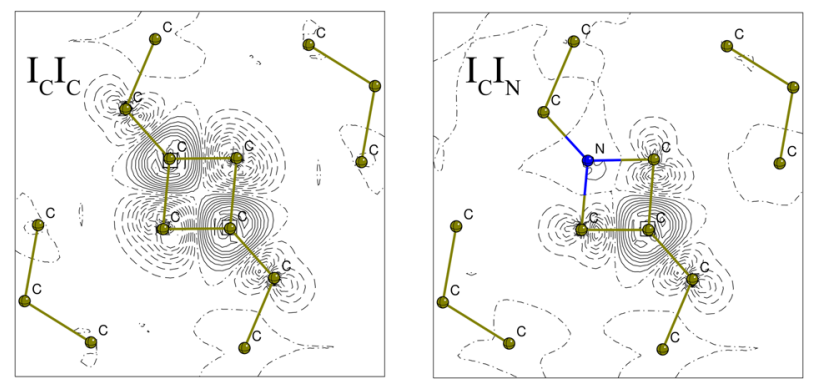

FIG. 4. Spin density maps for $I_{C} I_{C}$ and $I_{C} I_{N}$ in the plane through the four defect atoms that are coplanar. The spin density is truncated at $\pm 0.1|e| / a_{0}^{3}$, and the isodensity lines differ by $0.001|e| / a_{0}^{3}$. The continuous and dashed lines indicate positive and negative spin density values, respectively, while the dotted-dashed line indicates for zero spin density. The alternation of positive and negative spin density should be noticed, which propagates also to the neighbors of the defect atoms.
$1.03 \mathrm{eV}$. The empty spin-band of the unpaired electron is in the gap, $1.33 \mathrm{eV}$ below the $\mathrm{BCB}$, leading to an excitation energy of $4.56 \mathrm{eV}$ for the unpaired electron and one of $4.31 \mathrm{eV}$ for an excitation from the top of the valence band. The resulting local bandgap is $5.59 \mathrm{eV}$, a reduction of $0.14 \mathrm{eV}$ from the host lattice. Finally, as shown in the left panel of Fig. 3, in $\mathrm{I}_{C} \mathrm{I}_{C}$, the two unpaired $\mathrm{C}$ electrons occupy nearly degenerate spin states, split by $0.20 \mathrm{eV}, 1.00 \mathrm{eV}$ above the TVB, with the corresponding empty levels (one $\alpha$ and one $\beta$ ), separated by $0.42 \mathrm{eV}, \sim 1.11 \mathrm{eV}$ below the conduction band. This leads to a local narrowing of the bandgap to $5.65 \mathrm{eV}$.

\section{B. The hyperfine coupling tensor}

Our calculated EPR results for the four interstitial defects are reported in Table II, together with the experimental data, which have been attributed specifically to each of the defects. The first point to note is that the Fermi contact term, $A_{\text {iso }}$, and the components of the $B$ tensor for $\mathrm{I}_{C} \mathrm{I}_{C}(\mathrm{R} 1)$ and $\mathrm{I}_{C C}(\mathrm{R} 2)$ are much larger than those for the two other interstitials by up to two orders of magnitude.

In the $\mathrm{I}_{C} \mathrm{I}_{C}$ case (first line in Table II), the calculated values of $A_{i s o}, \mathrm{~B} 1, \mathrm{~B} 2$, and $\mathrm{B} 3$ are all close to the experimental values collected by Hunt et al., ${ }^{25}$ with a difference of $12 \%$ between the calculated $(+49 \mathrm{MHz})$ and experimental $(+43 \mathrm{MHz})$ values of the contact term and differences of $3.5 \%, 0.8 \%$, and $0.8 \%$ for the three principal components of $B$, where the largest of these is $+74 \mathrm{MHz}$ from the experiment compared with the simulated value of $+76 \mathrm{MHz}$. There is also very good agreement for the angles, $\theta$ and $\varphi$ (see Sec. II for definitions). It is worth noting that for this defect, there are no uncertainties as to the identity of the local structures responsible for the EPR measurements.

For the $\langle 100\rangle I_{C C}$ defect, the reported experimental data are very similar to those for $\mathrm{I}_{C} \mathrm{I}_{C}$, namely, $+78 \mathrm{MHz},-39 \mathrm{MHz}$, and 
TABLE II. Experimental and calculated isotropic and anisotropic components of the hyperfine coupling tensor. Expt. name indicates the name attributed by the experimental group to the defect. $\theta$ and $\varphi$ are the angles between the principal axis of the $B$ tensor and the $\langle 100\rangle$ and $\langle 001\rangle$ crystallographic directions. The results are for a $S_{216}$ supercell.

\begin{tabular}{|c|c|c|c|c|c|c|c|c|}
\hline Atom & $A_{\text {iso }}$ & $B_{1}$ & $B_{2}$ & $B_{3}$ & $\theta$ & $\varphi$ & Expt. name & References \\
\hline${ }^{13} \mathrm{C}$ in $\mathrm{I}_{\mathrm{C}} \mathrm{I}_{\mathrm{C}}$ & $\begin{array}{c}49.0 \\
+43.41\end{array}$ & $\begin{array}{c}73.9 \\
+76.47\end{array}$ & $\begin{array}{c}37.0 \\
-37.29\end{array}$ & $\begin{array}{c}37.0 \\
-39.18\end{array}$ & $\begin{array}{c}90 \\
90.00\end{array}$ & $\begin{array}{c}315 \\
314.67\end{array}$ & $\mathrm{R} 1$ & $\begin{array}{l}25 \text { and } 26 \\
\text { This work }\end{array}$ \\
\hline${ }^{13} \mathrm{C}$ in $\mathrm{I}_{\mathrm{CC}}$ & $\begin{array}{c}+44 \\
+25.38\end{array}$ & $\begin{array}{c}+78 \\
+76.34\end{array}$ & $\begin{array}{c}-39 \\
-38.66\end{array}$ & $\begin{array}{c}-39 \\
-37.68\end{array}$ & $\begin{array}{l}\text { ND } \\
90.00\end{array}$ & $\begin{array}{l}\text { ND } \\
90.00\end{array}$ & $\mathrm{R} 2$ & $\begin{array}{c}25 \\
\text { This work }\end{array}$ \\
\hline${ }^{15} \mathrm{~N}$ in $\mathrm{I}_{\mathrm{C}} \mathrm{I}_{\mathrm{N}}$ & $\begin{array}{c}0.00 \\
-2.55\end{array}$ & $\begin{array}{l}-1.01 \\
-1.40\end{array}$ & $\begin{array}{l}+1.00 \\
+0.87\end{array}$ & $\begin{array}{c}0.00 \\
+0.53\end{array}$ & $\begin{array}{l}134.82 \\
134.80\end{array}$ & $\begin{array}{l}45.00 \\
45.00\end{array}$ & WAR10 & $\begin{array}{c}27 \\
\text { This work }\end{array}$ \\
\hline${ }^{15} \mathrm{~N}$ in $\mathrm{I}_{\mathrm{CN}}$ & $\begin{array}{c}+8.10 \\
+14.30\end{array}$ & $\begin{array}{l}-0.25 \\
+1.77\end{array}$ & $\begin{array}{l}+0.20 \\
-1.74\end{array}$ & $\begin{array}{c}+0.07 \\
-0.034\end{array}$ & $\begin{array}{l}90.00 \\
89.98\end{array}$ & $\begin{array}{l}45.00 \\
45.00\end{array}$ & WAR9 & $\begin{array}{c}27 \\
\text { This work }\end{array}$ \\
\hline
\end{tabular}

$-39 \mathrm{MHz}$ compared with $+74 \mathrm{MHz},-37 \mathrm{MHz}$, and $-37 \mathrm{MHz}$, respectively, for the $B$ tensor and $44 \mathrm{MHz}$ as opposed to $49 \mathrm{MHz}$ for $A_{\text {iso }}$. There appears to be no reports for the angles, $\theta$ and $\varphi$, for $\mathrm{I}_{C C}$. Now, the computed values of the $\mathrm{I}_{C C} B$ tensor are very close to those of $\mathrm{I}_{C} \mathrm{I}_{C}$, with the largest component of $\mathrm{B}$, for example, $76 \mathrm{MHz}$ in both cases. The calculated values of $A_{i s o}$, on the other hand, are quite different, for the $\mathrm{I}_{C} \mathrm{I}_{C}$ value is $+43 \mathrm{MHz}$ and only $+25 \mathrm{MHz}$ in $\mathrm{I}_{C C}$, which is a difference of $53 \%$. There are two possible explanations for this apparent discrepancy. One is that the experimental data for the two defects are so close because they have been wrongly attributed and that, in fact, both sets of experiments refer to the same defect. The other is that the spectra of the two defects are indeed extremely similar and that the discrepancy between the experimental and simulated values of $A_{\text {iso }},+44 \mathrm{MHz}$ and $+25 \mathrm{MHz}$, respectively, is the result of inaccuracies in the calculations. We suggest that the first of these explanations is more likely for the following reasons:

- There is good agreement between the experimental and calculated values of $A_{\text {iso }}$ for $\mathrm{I}_{C} \mathrm{I}_{C}$, which, from the point of view of the present computational techniques per se, is similar to, though not, of course, identical to $\mathrm{I}_{C C}$, so that it is entirely reasonable to expect similar errors for the two defects.

- Hunt et al. have emphasized that all the experimental values are approximate because of the uncertainties in deconvoluting the various linewidths. ${ }^{45}$ For these reasons, we posit that the experimental results attributed to $\mathrm{I}_{C C}$ are, in fact, for $\mathrm{I}_{C} \mathrm{I}_{C}$.

Turning now to the heteronuclear double interstitial, $\mathrm{I}_{C} \mathrm{I}_{N}$, evaluating the EPR constants is difficult on two counts. The first is that the natural abundance of ${ }^{15} \mathrm{~N}$ is only $0.4 \%$; the second is that the comparatively large distance between $\mathrm{N}$ and the $\mathrm{C}$ atom bearing the unpaired electron, $1.86 \AA$, leads to constants whose absolute magnitudes are very small. Thus, an experimental value of zero has been reported for $A_{\text {iso }},{ }^{27}$ whereas the present calculations predict one of $-2.5 \mathrm{MHz}$. It is worth noting, however, that the absolute difference between the simulated and experimental values is smaller than that for $\mathrm{I}_{C} \mathrm{I}_{C}(6 \mathrm{MHz})$. The components of the $B$ tensor are, likewise, very small, but here, the measured and calculated constants are of similar magnitude and sign, with values of $-1.01 \mathrm{MHz}$ and $-1.40 \mathrm{MHz}$ for $\mathrm{B} 1,+1.00 \mathrm{MHz}$ and $+0.87 \mathrm{MHz}$ for $\mathrm{B} 2$, and $0.00 \mathrm{MHz}$ and $+0.53 \mathrm{MHz}$ for B3. The experimental and simulated angles, $\theta$ and $\varphi$, are also very similar. Thus, there appears to be good agreement between the calculated and observed constants, despite the very small values for both $A_{\text {iso }}$ and $B$.

The final defect we consider is $\mathrm{I}_{C N}$ for which the EPR constants are listed in the bottom row of Table II, where both the experimental and calculated values of the $B$ tensor are seen to be slightly larger than those of $\mathrm{I}_{C} \mathrm{I}_{N}$. However, unlike the latter defect, there are appreciable differences between the calculated and experimental constants, both in magnitude and sign, where the closest numbers, these for $\mathrm{B} 3$, are $-0.034 \mathrm{MHz}$ and $+0.07 \mathrm{MHz}$. One common feature is that the predicted value of $A_{i s o}$ and that deduced from experiment are both substantially larger than the components of $B$, which is unique among the four interstitial defects. The difference between the two values for $A_{i s o}$, where the prediction is $75 \%$ greater than experiment, together with the lack of agreement for any of the components of $B$, calls into doubt the attribution of the experimental constants to $\mathrm{I}_{C N}$.

\section{IR vibrational analysis}

The vibration spectrum of pristine diamond is particularly straight forward since, for reasons of symmetry, there are no IR active modes and just a single Raman peak at $1332 \mathrm{~cm}^{-1}, 46$ corresponding to the first-order scattering of triply degenerate $\mathrm{TO}(\mathrm{X})$ phonons of $t_{2 g}$. This compares with a value of $1317 \mathrm{~cm}^{-1}(-1 \%)$ derived from the B3LYP functional and modified Pople basis set here adopted. Therefore, all observed IR peaks and Raman peaks, other than that at $1332 \mathrm{~cm}^{-1}$, in the spectra of the three defective systems investigated here, can be assigned unambiguously to the presence of the impurity defects.

Experimental IR and Raman spectra have been collected by several authors cited above. ${ }^{25-27}$ However, for the most part, they have been used solely as a tool for determining the purity of samples rather than as an additional tool for the characterization of the defects, which has relied largely on interpretations of the EPR data. 
The IR spectra of the three defects considered here are shown in Fig. 5, where the vertical lines indicate the positions of the peaks of the single interstitial defects, namely, $\mathrm{I}_{C C}, \mathrm{I}_{N N}$, and $\mathrm{I}_{C N}$, which are included for comparison. The corresponding intensities and isotopic shifts are reported in Table III, while graphical animations of the modes are available at www.crystal.unito.it. Together, they represent key tools for the characterization of the various defect modes.

We start by analyzing the IR spectrum of $\mathrm{I}_{N} \mathrm{I}_{N}$, shown in the bottom panel of Fig. 5. Here, there are three dominant peaks at $1431 \mathrm{~cm}^{-1}, 1542 \mathrm{~cm}^{-1}$, and $1830 \mathrm{~cm}^{-1}$, with intensities of
$132 \mathrm{~km} / \mathrm{mol}, 314 \mathrm{~km} / \mathrm{mol}$, and $421 \mathrm{~km} / \mathrm{mol}$, respectively. There are also three other peaks in the lower wavenumber region of the spectrum at $341 \mathrm{~cm}^{-1}, 401 \mathrm{~cm}^{-1}$, and $553 \mathrm{~cm}^{-1}$, with intensities of $68 \mathrm{~km} / \mathrm{mol}, 51 \mathrm{~km} / \mathrm{mol}$, and $42 \mathrm{~km} / \mathrm{mol}$, respectively. All these peaks are related to the square planar part of the defective structure defined by $A, B, L$, and $M$, as the graphical animation confirms, while Table III indicates that the isotopic shift resulting from the change of mass from ${ }^{14} \mathrm{~N}$ to ${ }^{15} \mathrm{~N}$ is as large as $36 \mathrm{~cm}^{-1}$ and $19 \mathrm{~cm}^{-1}$ for the two highest wavenumber modes. By comparison, it is only $6 \mathrm{~cm}^{-1}$ for the mode at $341 \mathrm{~cm}^{-1}$ and negligible for the others. We posit that the

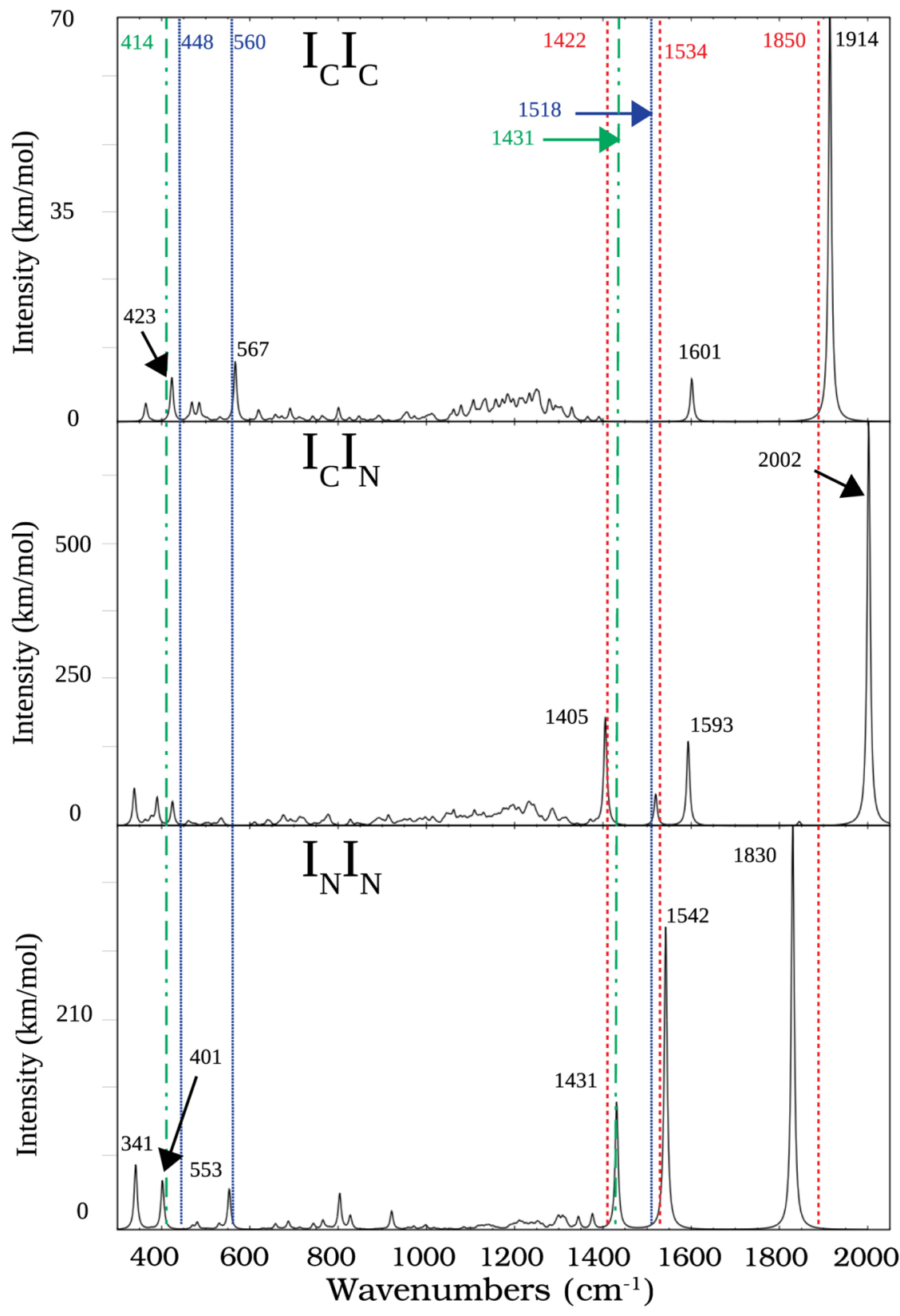

FIG. 5. Simulated IR spectrum of $I_{N} I_{N}$ (top), $I_{C} I_{N}$ (central), and $I_{C} I_{C}$ (bottom) defects. The vertical dashed lines indicate the relevant peaks of $I_{C C}$ (in blue) $I_{C N}$ (in red), and $I_{N N}$ (in green). The intensities of the peaks of both family defects are reported in Table III for better comparison, together with the shifts resulting from the isotopic substitution ${ }^{12} \mathrm{C}-{ }^{13} \mathrm{C}$ and ${ }^{14} \mathrm{~N}-{ }^{15} \mathrm{~N}$. 
TABLE III. Wavenumbers (in $\mathrm{cm}^{-1}$ ) and intensities (in $\mathrm{km} / \mathrm{mol}$, in brackets) of the six most intense normal modes of each defect, which are ordered from left to right by decreasing wavenumber. A, B, and L labels of atoms refer to Figs. 1 and 2. For each case, the effect of the isotopic substitution $(\mathrm{s})\left({ }^{12} \mathrm{C}-{ }^{13} \mathrm{C}\right.$ and $\left.{ }^{14} \mathrm{~N}-{ }^{15} \mathrm{~N}\right)$ is shown. The results are for the a $S_{216}$ supercell.

\begin{tabular}{|c|c|c|c|c|c|c|c|}
\hline Defect & Substitution & 1 & 2 & 3 & 4 & 5 & 6 \\
\hline \multirow{2}{*}{$\mathrm{I}_{\mathrm{C}} \mathrm{I}_{\mathrm{C}}$} & $\mathrm{B}, \mathrm{L}:{ }^{12} \mathrm{C}$ & $1914(69)$ & $1601(7)$ & $567(10)$ & $468(3)$ & $423(7)$ & $364(3)$ \\
\hline & B,L: ${ }^{13} \mathrm{C}$ & $1870(66)$ & $1577(7)$ & $564(9)$ & $468(3)$ & $419(8)$ & $362(4)$ \\
\hline \multirow{2}{*}{$I_{C C}$} & $\mathrm{~A}, \mathrm{~B}:{ }^{12} \mathrm{C}$ & $1519(10)$ & $1349(1)$ & $1338(3)$ & $1331(1)$ & $1280(2)$ & $448(7)$ \\
\hline & $\mathrm{A}, \mathrm{B}:{ }^{13} \mathrm{C}$ & $1490(9)$ & $1330(1)$ & $1337(4)$ & $1331(1)$ & $1280(2)$ & $442(8)$ \\
\hline \multirow{3}{*}{$\mathrm{I}_{\mathrm{C}} \mathrm{I}_{\mathrm{N}}$} & $\mathrm{B}, \mathrm{L}:{ }^{14} \mathrm{~N}^{12} \mathrm{C}$ & $2002(751)$ & $1844(8)$ & $1593(157)$ & $1405(198)$ & $425(45)$ & $390(51)$ \\
\hline & $\mathrm{B}:{ }^{15} \mathrm{~N}$ & $1974(804)$ & $1841(436)$ & $1533(272)$ & $1402(362)$ & $392(280)$ & $356(142)$ \\
\hline & $\mathrm{L}:{ }^{13} \mathrm{C}$ & 1945 (1158) & $1854(115)$ & $1532(250)$ & $1401(369)$ & $390(216)$ & $359(77)$ \\
\hline \multirow{3}{*}{$\mathrm{I}_{\mathrm{CN}}$} & B,L: ${ }^{14} \mathrm{~N}^{12} \mathrm{C}$ & $1850(265)$ & $1534(86)$ & $1422(302)$ & $548(11)$ & $406(41)$ & $360(31)$ \\
\hline & $\mathrm{B}:{ }^{15} \mathrm{~N}$ & $1823(254)$ & $1534(86)$ & $1400(280)$ & $547(9)$ & $402(34)$ & $354(38)$ \\
\hline & $\mathrm{L}:{ }^{13} \mathrm{C}$ & $1813(260)$ & $1504(80)$ & $1422(302)$ & 547 (11) & $406(41)$ & $359(30)$ \\
\hline \multirow{2}{*}{$\mathrm{I}_{\mathrm{N}} \mathrm{I}_{\mathrm{N}}$} & $\mathrm{B}, \mathrm{L}:{ }^{14} \mathrm{~N}$ & $1830(421)$ & $1542(314)$ & $1431(132)$ & $341(68)$ & $401(51)$ & $553(42)$ \\
\hline & B,L: ${ }^{15} \mathrm{~N}$ & $1794(405)$ & $1523(250)$ & $1431(131)$ & $335(74)$ & $399(44)$ & $552(38)$ \\
\hline \multirow{2}{*}{$\mathrm{I}_{\mathrm{NN}}$} & $\mathrm{A}, \mathrm{B}:{ }^{14} \mathrm{~N}$ & $1431(350)$ & $1354(32)$ & $1346(23)$ & $550(29)$ & $414(102)$ & $364(44)$ \\
\hline & $\mathrm{A}, \mathrm{B}:{ }^{15} \mathrm{~N}$ & 1407 (327) & $1347(27)$ & $1346(27)$ & $549(24)$ & $409(89)$ & $360(58)$ \\
\hline
\end{tabular}

high intensity of the defect peaks can be attributed to the large polarity of the bonds involving the $\mathrm{N}$ atoms. Now, the $\mathrm{I}_{N} \mathrm{I}_{N}$ spectrum can be compared with those of the simpler defects, $\mathrm{I}_{N N}$ and $\mathrm{I}_{C N}$, both of which contain nitrogen. The IR spectrum of $\mathrm{I}_{N N}$ is characterized by only two intense peaks (see Table III). The first at $1431 \mathrm{~cm}^{-1}$, with an intensity of $350 \mathrm{~km} / \mathrm{mol}$, is exactly the same as that of an $\mathrm{I}_{N} \mathrm{I}_{N}$ mode of E symmetry; the second at $414 \mathrm{~cm}^{-1}$, with an intensity of $102 \mathrm{~km} / \mathrm{mol}$, is just $13 \mathrm{~cm}^{-1}$ above one of the $\mathrm{I}_{N} \mathrm{I}_{N}$ peaks, again of $\mathrm{E}$ symmetry. In $\mathrm{I}_{C N}$, the lower $\left(\mathrm{C}_{2 v}\right)$ symmetry activates a $\mathrm{CN}$ symmetric stretching mode at $1850 \mathrm{~cm}^{-1}$, with an intensity of $265 \mathrm{~km} / \mathrm{mol}$, which is merely $20 \mathrm{~cm}^{-1}$ above the highest peak in the $\mathrm{I}_{N} \mathrm{I}_{N}$ spectrum. In addition, the $\mathrm{E}$ mode of the more symmetric $\mathrm{I}_{N N}$ splits into two, giving one at $1534 \mathrm{~cm}^{-1}(86 \mathrm{~km} / \mathrm{mol})$ and one at $1422 \mathrm{~cm}^{-1}$ $(120 \mathrm{~km} / \mathrm{mol})$, which are quite close to the modes at $1542 \mathrm{~cm}^{-1}$ and $1431 \mathrm{~cm}^{-1}$ of $\mathrm{I}_{N} \mathrm{I}_{N}$.

In summary, the three dominant peaks of $\mathrm{I}_{N} \mathrm{I}_{N}$ are extremely close to those of $\mathrm{I}_{C N}$, with differences $9 \mathrm{~cm}^{-1}, 8 \mathrm{~cm}^{-1}$, and $20 \mathrm{~cm}^{-1}$, and to two of the modes of $\mathrm{I}_{N N}$, with differences $0 \mathrm{~cm}^{-1}$ and $13 \mathrm{~cm}^{-1}$.

The symmetric stretch of $\mathrm{I}_{C N}$ at $1850 \mathrm{~cm}^{-1}$ increases to $2002 \mathrm{~cm}^{-1}$ in $\mathrm{I}_{C} \mathrm{I}_{N}$, while the intensity increases from $265 \mathrm{~km} / \mathrm{mol}$ in $I_{C N}$ to $751 \mathrm{~km} / \mathrm{mol}$, which is the most intense of all the defects examined here. The two other important peaks are at $1405 \mathrm{~cm}^{-1}$ and $1593 \mathrm{~cm}^{-1}$, with intensities of $198 \mathrm{~km} / \mathrm{mol}$ and $157 \mathrm{~km} / \mathrm{mol}$, respectively. The first is $26 \mathrm{~cm}^{-1}$ below, and the second is $51 \mathrm{~cm}^{-1}$ above the corresponding peak of $\mathrm{I}_{N} \mathrm{I}_{N}$. Furthermore, it is of interest to note that the $1405 \mathrm{~cm}^{-1}$ peak occurs in a similar region to those of both $\mathrm{I}_{N N}$ and $\mathrm{I}_{C N}$. A fourth, low intensity absorption is predicted at $1519 \mathrm{~cm}^{-1}$, which is close to those of the two single defects at $1518 \mathrm{~cm}^{-1}\left(\mathrm{I}_{C C}\right)$ and $1534 \mathrm{~cm}^{-1}\left(\mathrm{I}_{C N}\right)$.
The predicted spectrum of $\mathrm{I}_{C} \mathrm{I}_{C}$ is simple, with a single peak of moderate intensity $(69 \mathrm{~km} / \mathrm{mol})$ at $1914 \mathrm{~cm}^{-1}$, which is intermediate between those of $\mathrm{I}_{C} \mathrm{I}_{N}$ at $1830 \mathrm{~cm}^{-1}$ and $\mathrm{I}_{N} \mathrm{I}_{N}$ at $2002 \mathrm{~cm}^{-1}$. There are also three peaks with much lower intensities. They occur at $1601 \mathrm{~cm}^{-1}(7 \mathrm{~km} / \mathrm{mol})$, which is $8 \mathrm{~cm}^{-1}$ above the $1593 \mathrm{~cm}^{-1}$ $\mathrm{I}_{C} \mathrm{I}_{N}$ peak, at $567 \mathrm{~cm}^{-1}(\mathrm{I}=10 \mathrm{~km} / \mathrm{mol}$, which is close to a similar peak of $\left.\mathrm{I}_{N} \mathrm{I}_{N}\right)$, and at $423 \mathrm{~cm}^{-1}(\mathrm{I}=7 \mathrm{~km} / \mathrm{mol})$. The isotopic shift is as large as $44 \mathrm{~cm}^{-1}$ for the mode at $1914 \mathrm{~cm}^{-1}$ and $24 \mathrm{~cm}^{-1}$ for the mode at $1601 \mathrm{~cm}^{-1}$ but negligible for the other modes. It is important to note the very different intensity scales in Fig. 5 for the three defects. For interstitial defects with only carbon atom, the polarization of the $\mathrm{AB}$ LM bonds is much lower, and the intensities are up to 10 times smaller than for the other defects. The IR spectrum of the third, single interstitial defect, $\mathrm{I}_{C C}$, is predicted to contain peaks with very low intensities for the reasons discussed above. The peak at $448 \mathrm{~cm}^{-1}$ has an intensity of only $14 \mathrm{~km} / \mathrm{mol}$ and that at $1531 \mathrm{~cm}^{-1}$ has an intensity of $20 \mathrm{~km} / \mathrm{mol}$ so that these peaks are unlikely to be detected in the experimental spectrum with any clarity or precision.

\section{DISCUSSION AND CONCLUSIONS}

The principal purpose of this paper is to report, for the first time, all-electron calculations of the Fermi contact term, $A_{\text {iso }}$, and components of the hyperfine coupling tensor, $B$, of two single interstitial defects, $\mathrm{I}_{C C}$ and $\mathrm{I}_{C N}$, and two double interstitial defects, $\mathrm{I}_{C} \mathrm{I}_{N}$ and $\mathrm{I}_{C} \mathrm{I}_{C}$ (Fig. 1), and to compare these with the reported EPR data. ${ }^{25-27}$ Of particular interest are the systems where there are marked disparities between the predicted constants and those derived from the EPR measurements, for which a reasoned 
explanation is suggested. Also reported are the IR spectra of the double interstitials, Raman spectra being less useful in view of their very low intensity by comparison with the pristine diamond peak at $1317 \mathrm{~cm}^{-1}$. In addition and for the purpose of exact comparison, the EPR constants of the single $\mathrm{I}_{C N}$ and $\mathrm{I}_{C C}$ interstitial defects (Fig. 2), whose electronic structures and IR spectra have been reported previously, ${ }^{1,30}$ have also been included in this study.

As a preliminary comment, we note that IR spectra are able to provide, at least, in principle, information on all six defects, whereas EPR can only be used to study open shell systems so that both $\mathrm{I}_{N N}$ and $\mathrm{I}_{N} \mathrm{I}_{N}$ are excluded. However, a useful way to by-pass this limitation is to generate the corresponding charged defects, whose ground states might turn out to be open shell, as is the case with $\mathrm{VN}_{2}^{-}$and $\mathrm{N}_{2}^{+}$(see Refs. 32 and 33).

We start with $\mathrm{I}_{C} \mathrm{I}_{C}$, for which, as Table II indicates, there is excellent agreement with experiment for the three distinct sets of constants that are obtained from the EPR data, namely, the Fermi contact term, the eigenvalues of the hyperfine coupling tensor $B$, and the angles, $\theta$ and $\varphi$, which define the orientation of the principal axes of $B$ with respect to the crystallographic axes. This close agreement between the calculated and observed values of six EPR constants attests to the efficacy of the computational methods used in this study. However, perhaps of greater significance in the context of this study is that it leaves no ambiguity in attributing the reported measured constants to the predicted lattice and electronic structures we have assigned to $\mathrm{I}_{C} \mathrm{I}_{C}$. This verification is particularly useful, for the IR spectrum is characterized by peaks of very low intensity since the dipole moments generated by the defect are extremely small. Thus, in spectra of systems containing other defects such as $\mathrm{N}$, which is nearly always present, the $\mathrm{I}_{C} \mathrm{I}_{C}$ peaks would be swamped and certainly not sufficiently visible for identification purposes.

As shown in Table II, the experimental EPR data reported in Ref. 25, purportedly for the single interstitial defect, I $C C$, are very close to that for $\mathrm{I}_{C} \mathrm{I}_{C}$, but only in part. For while there is very good agreement with our predicted values of the hyperfine coupling tensor, $B$, for the two interstitial defects, with an average deviation across the three components of $2 \%$, the values of the Fermi contact term, $A_{\text {iso }}$, are quite different, with an observed value of $44 \mathrm{MHz}$ compared with a prediction of $25.4 \mathrm{MHz}$. Since the double interstitial is the more stable of the two defects, it is entirely possible that samples of $I_{C C}$ might be contaminated by low concentrations of $\mathrm{I}_{C} \mathrm{I}_{C}$ so that the EPR spectrum attributed to $\mathrm{I}_{C C}$ by Hunt ${ }^{25}$ is, in fact, that of $\mathrm{I}_{C} \mathrm{I}_{C}$. Our calculations would certainly support this possibility, which new EPR experiments might help to clarify. In the absence of such data, the IR spectra might be useful here, for the $I_{C C} 1518 \mathrm{~cm}^{-1}$ peak, though weak $(10 \mathrm{~km} / \mathrm{mol})$, is separated by $83 \mathrm{~cm}^{-1}$ from that at $1601 \mathrm{~cm}^{-1}$ in the $\mathrm{I}_{C} \mathrm{I}_{C}$ spectrum, which is also weaker.

We note that the IR spectrum is of little value here for the confirming the presence of $I_{C C}$ since all the predicted defect peaks have even lower intensities, $10 \mathrm{~km} / \mathrm{mol}$ or less, than those for $\mathrm{I}_{C} \mathrm{I}_{C}$, the least intense of which is calculated to be $69 \mathrm{~km} / \mathrm{mol}$. Turning now to the N-containing interstitials, as shown in Fig. 2 (b), the distance between the $\mathrm{N}$ atom (B) and the $\mathrm{C}$ atom with the unpaired spin (L) in $\mathrm{I}_{C} \mathrm{I}_{N}$ is appreciably larger than the normal lattice spacing, leading to a weak spin-spin interaction with the ${ }^{15} \mathrm{~N}$ nucleus. This is reflected in the very small values of both the measured and calculated values of $A_{\text {iso }}$ and $B$, as indicated in Table II, where the differences between these for the components of $B$ are comparable to those for $\mathrm{I}_{C} \mathrm{I}_{C}$ and $\mathrm{I}_{C C}$. Unlike the pure carbon interstitials, no such ambiguity can arise in the case of $\mathrm{I}_{C} \mathrm{I}_{N}$ as to the attribution of the EPR constants, since $\mathrm{I}_{N N}$ is spin inactive. In view of this, any uncertainty in identifying the experimental EPR spectrum with the microscopic structure that we indicate as $\mathrm{I}_{C} \mathrm{I}_{N}$ is much less certain. It might also be worth noting that in the absence of isotope enrichment, the natural abundance of ${ }^{13} \mathrm{C}$ is $\sim 30$ times that of ${ }^{15} \mathrm{~N}$. What might clarify this issue is the IR spectrum, for, as Fig. 5 indicates, the $\mathrm{I}_{C} \mathrm{I}_{N}$ spectrum contains an intense $(751 \mathrm{~km} / \mathrm{mol})$ peak at $2002 \mathrm{~cm}^{-1}$, which is separated by $90 \mathrm{~cm}^{-1}$ from all the other interstitial peaks. To date, there are no reports of the experimental spectrum.

In the case of $\mathrm{I}_{C N}$, we find very substantial differences between the predicted values of $A_{\text {iso }}$ and $B$ and those reported by Felton et al. from their EPR data, with errors of $55 \%$ for $A_{\text {iso }}$ and over $200 \%$ for the $B_{1}$ and $B_{2}$ components of $B$. In view of the good accord for other systems and the reliability of our computational procedures, more generally, we suggest that the experimental results might not relate to the $\mathrm{I}_{C N}$ structure we have derived in this study. Here again, the IR spectrum is of potential value in characterizing this defect, for the predicted intense $(265 \mathrm{~km} / \mathrm{mol})$ peak at $1850 \mathrm{~cm}^{-1}$ is sufficiently separated from that of $\mathrm{I}_{N} \mathrm{I}_{N}$ at $1830 \mathrm{~cm}^{-1}(421 \mathrm{~km} / \mathrm{mol})$ and well separated from those of the other defects. The final two interstitials we have examined are $\mathrm{I}_{N N}$ and $\mathrm{I}_{N} \mathrm{I}_{N}$, which have closed shell ground states, and are, consequently, EPR inactive. The only recourse to the hyperfine coupling constants, therefore, would be via their charged states, which, to date, appear not to have been examined. However, there are major differences in the predicted IR spectra of the two interstitials, which might be exploited. The predicted $\mathrm{I}_{N} \mathrm{I}_{N}$ spectrum contains two intense peaks, one at $1830 \mathrm{~cm}^{-1}(\mathrm{I}=421 \mathrm{~km} / \mathrm{mol})$ and the other at $1541(\mathrm{I}=314 \mathrm{~km} / \mathrm{mol})$, whereas the spectrum of $\mathrm{I}_{N N}$ exhibits a single strong peak at $1431 \mathrm{~cm}^{-1}(\mathrm{I}=350 \mathrm{~km} / \mathrm{mol})$. Since the latter is roughly 20 times the intensity of the peak in the $\mathrm{I}_{N} \mathrm{I}_{N}$ spectrum with exactly the same frequency, there would seem to be little by way of confusion. $I_{C N}$ has a peak at $1422 \mathrm{~cm}^{-1}$, but these can be distinguished using ${ }^{15} \mathrm{~N}$ isotopic shifts. Again, to date, there appears to be no experimental IR data for these defects or for $\mathrm{I}_{C N}$.

In summary, this study has shown that spectroscopies such as IR and EPR, while extremely informative for some defect systems, when used singly, can be restricted in the information they are able to provide for others. On the other hand, the combination of IR, EPR, and simulations, based on high-level quantum mechanical calculations, can provide a comprehensive, unified, and consistent description of the microscopic structural, dynamic, and electronic properties of a much wider range of materials.

The specific conclusions of this study are as follows:

- $\mathrm{I}_{C} \mathrm{I}_{C}$ can be uniquely identified by its observed EPR constants.

- The close similarity of the reported experimental values of the anisotropic term, $\mathbf{B}$, of $\mathrm{I}_{C C}$ and $\mathrm{I}_{C} \mathrm{I}_{C}$, in conjunction with the calculations reported here, raises questions as to the correct attribution of the EPR data for $\mathrm{I}_{C C}$.

- The very low values of the experimental and predicted EPR constants of $\mathrm{I}_{C} \mathrm{I}_{N}$, which result from weak spin-spin interactions, for structural reasons, suggest that these alone do not 
provide strong evidence for the identity of this defect and that the experimental IR spectrum would make an important contribution to this.

- The experimental EPR constants of $I_{C N}$ might not relate to the lattice and electronic structures we have calculated for this defect, but the reliable IR data might clarify this issue.

- In the absence of charged species of $\mathrm{I}_{N N}$ and $\mathrm{I}_{N} \mathrm{I}_{N}$, from which EPR constants might be obtained, the differences in their predicted IR spectra suggest that the experimental spectra could provide a useful and accurate means of identification.

\section{ACKNOWLEDGMENTS}

F.S.G. acknowledges the CINECA award under the ISCRA initiative (Grant No. HP10BJO47B) for the availability of high performance computing resources and support. A.P. is thankful for the financial support from EUROfusion Enabling Research Project No. ENR-MFE19.ISSP-UL-02 "Advanced experimental and theoretical analysis of defect evolution and structural disordering in optical and dielectric materials for fusion application." The views and opinions expressed herein do not necessarily reflect those of the European Commission.

\section{DATA AVAILABILITY}

The data that support the findings of this study are available within the article.

\section{REFERENCES}

${ }^{1}$ S. Salustro, F. Pascale, W. C. Mackrodt, C. Ravoux, A. Erba, and R. Dovesi, Phys. Chem. Chem. Phys. 20, 16615 (2018).

${ }^{2}$ J. P. Bade, S. R. Sahaida, B. R. Stoner, J. A. Von Windheim, J. T. Glass, K. Miyata, K. Nishimura, and K. Kobashi, Diamond Relat. Mater. 2, 816 (1993).

${ }^{3}$ P. Bergonzo, D. Tromson, and C. Mer, J. Synchrotron Radiat. 13, 151 (2006).

${ }^{4}$ G. Aiello, T. Scherer, K. Avramidis, N. Casal, T. Franke, M. Gagliardi, G. Gantenbein, M. Henderson, J. Jelonnek, A. Meier et al., Fusion Sci. Technol. 75, 719 (2019).

${ }^{5}$ S. J. Breuer and P. R. Briddon, Phys. Rev. B 51, 6984 (1995).

${ }^{6}$ F. Gentile, S. Salustro, G. Di Palma, M. Causá, P. D’arco, and R. Dovesi, Theor. Chem. Acc. 137, 154 (2018).

${ }^{7}$ A. Mainwood, Diamond Relat. Mater. 8, 1560 (1999).

${ }^{8}$ J. P. Goss, B. J. Coomer, R. Jones, C. J. Fall, P. R. Briddon, and S. Öberg, Phys. Rev. B 67, 165208 (2003).

${ }^{9}$ S. Salustro, F. Colasuonno, A. M. Ferrari, M. D'Amore, W. C. Mackrodt, and R. Dovesi, Carbon 146, 709 (2019).

${ }^{10}$ D. J. Twitchen, D. C. Hunt, M. E. Newton, J. M. Baker, T. R. Anthony, and W. F. Banholzer, Phys. B: Condens. Matter 273-274, 628 (1999).

${ }^{11}$ H. Amekura and N. Kishimoto, J. Appl. Phys. 104, 063509 (2008).

${ }^{12}$ D. N. Jamieson, S. Prawer, K. W. Nugent, and S. P. Dooley, Phys. Rev. B 106, 641 (1995).
${ }^{13}$ A. Gali, Phys. Rev. B 79, 235210 (2009).

${ }^{14}$ T. Schröder, M. E. Trusheim, M. Walsh, L. Li, J. Zheng, M. Schukraft, A. Sipahigil, R. E. Evans, D. D. Sukachev, C. T. Nguyen et al., Nat. Commun. 8, 15376 (2017).

${ }^{15}$ A. Mainwood, J. Phys. C: Solid State Phys. 11, 2703 (1978).

${ }^{16}$ U. Gerstmann, M. Amkreutz, and H. Overhof, Phys. Rev. B 60, R8446 (1999).

${ }^{17}$ J. A. van Wyk, O. D. Tucker, M. E. Newton, J. M. Baker, G. S. Woods, and P. Spear, Phys. Rev. B 52, 12657 (1995).

${ }^{18}$ A. Zelferino, S. Salustro, J. Baima, V. Lacivita, R. Orlando, and R. Dovesi, Theor. Chem. Acc. 135, 74 (2016).

${ }^{19}$ J. P. Goss, B. J. Coomer, R. Jones, T. D. Shaw, P. R. Briddon, M. Rayson, and S. Öberg, Phys. Rev. B 63, 195208 (2001).

${ }^{20}$ G. Davies, H. Smith, and H. Kanda, Phys. Rev. B 62, 1528 (2000).

${ }^{21}$ J. P. Goss, R. Jones, T. D. Shaw, M. J. Rayson, and P. R. Briddon, Phys. Status Solidi A 186, 215 (2001).

${ }^{22}$ M. E. Newton, B. A. Campbell, D. J. Twitchen, J. M. Baker, and T. R. Anthony, Diamond Relat. Mater. 11, 618 (2002).

${ }^{23}$ S. Salustro, Y. Nöel, C. M. Zicovich-Wilson, P. Olivero, and R. Dovesi, J. Chem. Phys. 145, 184701 (2016).

${ }^{24}$ O. D. Tucker, M. E. Newton, and J. M. Baker, Phys. Rev. B 50, 15586 (1994).

${ }^{25}$ D. Hunt, D. Twitchen, M. Newton, J. Baker, J. Kirui, J. van Wyk, T. Anthony, and W. Banholzer, Phys. Rev. B 62, 6587 (2000).

${ }^{26}$ D. J. Twitchen, M. E. Newton, J. M. Baker, O. D. Tucker, T. R. Anthony, and W. F. Banholzer, Phys. Rev. B 54, 6988 (1996).

${ }^{27}$ S. Felton, B. L. Cann, A. M. Edmonds, S. Liggins, R. J. Cruddace, M. E. Newton, D. Fisher, and J. M. Baker, J. Phys.: Condens. Matter 21, 364212 (2009).

${ }^{28}$ R. Dovesi, A. Erba, R. Orlando, C. M. Zicovich-Wilson, B. Civalleri, L. Maschio, M. Rérat, S. Casassa, J. Baima, S. Salustro et al., Wires 8, e1360 (2018).

${ }^{29}$ R. Dovesi, V. R. Saunders, C. Roetti, R. Orlando, C. M. Zicovich-Wilson, F. Pascale, B. Civalleri, K. Doll, N. M. Harrison, I. J. Bush et al., CRYSTAL 2017 User's Manual (University of Torino, Torino, 2017).

${ }^{30}$ S. Salustro, A. Erba, C. M. Zicovich-Wilson, Y. Nöel, L. Maschio, and R. Dovesi, Phys. Chem. Chem. Phys. 18, 21288 (2016).

${ }^{31}$ A. M. Ferrari, S. Salustro, F. S. Gentile, W. C. Mackrodt, and R. Dovesi, Carbon 134, 354 (2018)

${ }^{32}$ G. Di Palma, B. Kirtman, F. S. Gentile, A. Platonenko, A. M. Ferrari, and R. Dovesi, Carbon 159, 443 (2020).

${ }^{33}$ G. Di Palma, F. S. Gentile, V. Lacivita, W. Mackrodt, R. Dovesi et al., J. Mater. Chem. C 8, 5239 (2020).

${ }^{34}$ A. D. Becke, J. Chem. Phys. 98, 5648 (1993).

${ }^{35}$ C. Lee, W. Yang, and R. G. Parr, Phys. Rev. B 37, 785 (1988).

${ }^{36}$ J. S. Binkley, J. A. Pople, and W. J. Hehre, J. Am. Chem. Soc. 102, 939 (1980).

${ }^{37}$ H. Kjær and S. S. P. Sauer, J. Chem. Theory Comput. 7, 4070 (2011).

${ }^{38}$ A. D. Becke, J. Chem. Phys. 88, 2547 (1988).

${ }^{39}$ H. J. Monkhorst and J. D. Pack, Phys. Rev. B 13, 5188 (1976).

${ }^{40}$ F. Pascale, C. M. Zicovich-Wilson, F. López Gejo, B. Civalleri, R. Orlando, and R. Dovesi, J. Comput. Chem. 25, 888 (2004).

${ }^{41}$ S. Dall'Olio, R. Dovesi, and R. Resta, Phys. Rev. B 56, 10105 (1997).

${ }^{42}$ R. D. King-Smith and D. Vanderbilt, Phys. Rev. B 49, 5828 (1994).

${ }^{43}$ H. E. Smith, G. Davies, M. E. Newton, and H. Kanda, Phys. Rev. B 69, 045203 (2004).

${ }^{44}$ M. Cardona and M. L. W. Thewalt, Rev. Mod. Phys. 77, 1173 (2005).

${ }^{45}$ D. C. Hunt, D. J. Twitchen, M. E. Newton, J. M. Baker, T. R. Anthony, W. F. Banholzer, and S. S. Vagarali, Phys. Rev. B 61, 3863 (2000).

${ }^{46}$ S. Prawer and R. J. Nemanich, Philos. Trans. R. Soc., A 362, 2537 (2004). 